\title{
Development of a haptic feedback actuator for an endovascular interventions simulator interface
}

\begin{abstract}
Endovascular interventions are minimally invasive surgical procedures that are performed to diagnose and treat vascular diseases using flexible instruments known as guidewire and catheter. A popular method of developing the skills required to manipulate the instruments successfully is through the use of virtual reality (VR) simulators. However, the interfaces of current VR simulators have several shortcomings and a major challenge of addressing the shortcomings is to unobtrusively access the central, co-axial guidewire for haptics. This work sets out to explore two different approaches for applying force to the concentric guidewire: direct non-contact electromagnetic force application and a custom actuator designed to be embedded within a catheter hub. An initial implementation of the hub actuator approach was able to produce a suitably strong resistance effect when the guidewire is advanced, but further work is needed to reduce the actuator size while maintaining the level of resistance.
\end{abstract}

